\title{
Vendor qualification: Utilization of solid state characterization "Toolbox" to assess material variability for active pharmaceutical ingredient
}

\author{
Devarajan Saravanan ${ }^{1 *}$, Prakash Muthudoss ${ }^{1}$, Praveen Khullar $^{1}$, Ambrose Rosevenis ${ }^{2}$ \\ ${ }^{1}$ Sanofi Synthelabo India Private Limited, Verna, India. \\ ${ }^{2}$ Department of Chemistry, St. Josephs College, Tiruchirappalli, India.
}

\section{ARTICLE INFO \\ Received on: 08/05/2019 \\ Accepted on: 12/07/2019 \\ Available online: 01/09/2019}

\section{Key words:}

Vendor qualification, drug product performance testing, solid state characterization techniques, in situ processinduced fragmentation, critical material attributes.

\begin{abstract}
One of the credos for a successful product development, early clinical trial supplies, achieving full-scale manufacturability and speed to the market is the vendor qualification. The focus of this paper is to employ a systematic approach to qualify different active pharmaceutical ingredient (API) suppliers. In this context, API sourced from two different vendors used in product development, where prototype formulations manufactured with identical components and specifications demonstrated significant variations in drug product performance attributable to vendor-to-vendor variability. Prototype prepared using API (Vendor 1) showed disintegration of tablets in 4.5 minutes which complied with in-house specifications, whereas it was $>15$ minutes for the prototype prepared from API (Vendor 2). In order to understand these differences, a vast array of solid state techniques were employed to compare the critical material attributes of API (GDCS1902) from two different Vendors. Furthermore, these tools were orthogonally applied to understand whether API from two Vendors demonstrated any process-induced transformations, such as processinduced polymorphism, process-induced crystal disorder, and process-induced fragmentation. The results of these measurements indicated the presence of fine particles of varied morphology with API (Vendor 1), while API (Vendor 2) showed more medium-sized uniform particles. Formulation process modification to induce API fragmentation in situ was carried out for the API from Vendor 2. This modification produced desired granule properties which were then subjected to drug performance tests and was found to match the specification. This study demonstrates the importance of understanding the critical material attributes to match the final product performance when multiple vendors were selected.
\end{abstract}

\section{INTRODUCTION}

Oral route is the most frequently used route of drug administration and tablets are the most common solid oral dosage form. A tablet is not just a pressed block of single material but a complex matrix containing one or more active pharmaceutical ingredients (APIs) and other excipients that are considered as raw materials (RM). Quality assurance of RM includes setting appropriate specification and control of these components that are of substantial importance during manufacturing (Elder et al., 2016).

*Corresponding Author

D. Saravanan, Sanofi Synthelabo India Private Limited, Verna, India. E-mail:d.saravanan@sanofi.com
The raw material specifications should meet the expectations of Regulatory authorities or the National Pharmacopoeias or be in line with ICH guidelines. At times API complying with specifications has resulted in unexpected finished product performance. In such cases, certificate of analysis to demonstrate the equivalency between vendors and/or batches is inadequate (Kushner, 2013).

In order to assure the quality of finished dosage formulations, the RM qualification and control program should be considered as a key factor and a step to achieve, right firsttime and right every-time approach. However, time and again RM qualification is deemed of secondary importance. Raw material testing is not considered scientifically exciting unless the supply chain/single vendor/quality of RM/facility audits are put at risk (Zarmpi et al., 2017). To circumvent any such risks, chemical and physical properties of the desired RM and their suppliers 
should be thoroughly investigated and qualified both initially and periodically (Elder et al., 2016; Kushner, 2013; Zarmpi et al., 2017).

The work presented is a part of an investigation carried out to understand critical material attributes of RM which could be an invincible approach to select different vendors as part of quality or commercial needs. Knowledge of structural and chemical properties of the active ingredient(s) and excipients is essential for successful product development (Stauffer et al., 2018). Unfortunately, there is no "one size fits all" analytical method for approaching these complex analyses; hence, a suite of solid-state analytical tools have been utilized to complement GDCS1902 particle, molecular, and bulk level properties (Byrn et al., 2017).

Powder X-ray Diffraction (PXRD) is sensitive to long-range order of crystal lattice while Raman spectroscopy provides short-range intermolecular interactions and molecular conformations (Byrn et al., 2017; Chennuru et al., 2016; Paudel et al., 2015). Thermo-analytical techniques (Giron, 2013; Newman and Wenslow, 2018), such as Differential Scanning Calorimetry (DSC) and Thermogravimetric analysis, provides additional information about the API's physical or chemical stability, thermodynamic properties, and to an extent, the physicochemical properties of the RM. Polarized Light Microscopy (PLM) provides optical crystallographic information which is sensitive to organic small molecules' orientation and conformation (Carlton, 2011). On the other hand, Near-Infrared (NIR) spectroscopy (Kandpal et al., 2015; Moros et al., 2010; Pasquini, 2011) can provide simultaneous physical and chemical property correlation which at times is very useful as both black-box and white-box analytical tool. However, each of these methods has its own pros and cons but correlation displayed between the results provides indispensable information to the formulation team.

\section{BACKGROUND}

Vendor selection and qualification is considered as an integral part of pharmaceutical product development. Selection of at least two different vendors should be a part of the raw material procurement process. In this context, GDCS1902 from two different Vendors (Vendor 1 and Vendor 2) were procured and two prototype formulations were prepared. Prototype 1 and Prototype 2 were prepared using API from Vendor 1 and Vendor 2, respectively. The in-house specification limit for the disintegration time is $<5$ minutes and drug product release is $>70 \%$ in 45 minutes (single point dissolution). When subjected to drug product performance testing, these two prototypes were found to be dissimilar with respect to drug product disintegration, that is, the disintegration time for prototype formulation 1 and 2 were $\sim 4.5$ minutes and $\sim 15$ minutes, respectively. In order to evaluate this dissimilarity between formulations, a vast array of solid state analytical tools in combination with chemometrics were employed to unravel the material properties of GDCS1902. The approach of integrating drug substance critical material attributes (CMAs) with manufacturing process provides a better understanding of the drug product development (Docherty et al., 2009; Ferreira et al., 2018; Schenck et al., 2019; Stauffer et al., 2019; Zhao et al., 2019). The selection of manufacturing process for drug substance by different vendors (crystallization, selection of solvents, drying, particle size reduction like milling, micronizing, etc.) could determine the physical properties and the final raw material attributes of GDCS1902 (Docherty et al., 2009; Ferreira et al., 2018). Considering this extensive API, raw material characterization was carried out to discriminate the attributes of API between Vendor 1 and Vendor 2 for process-induced transformations like process-induced polymorphism (PIP) (Elisei et al., 2018; Ho et al., 2012; Trasi et al., 2010), process-induced crystal disorder (PICD) (Descamps and Willart, 2016; Zimper et al., 2010), or process-induced Fragmentation (PIF) (Zhao et al., 2019). A Modified formulation (Prototype 3) was prepared based on the studies of raw material characteristics.

\section{MATERIALS AND METHODS}

\section{Materials}

Model API coded as GDCS1902 from two different Vendors (Vendor 1 and Vendor 2) were used as received. The physical properties of the API are included in Table 1. Furthermore, studies were carried out using tablets to understand the impact of two different API on the product performance The composition of prototypes used in this study were same (API 40\% and excipients $60 \%$ ). Prototype 1 and Prototype (2 and 3) were manufactured through wet granulation using API (Vendor 1) and Vendor 2, respectively.

\section{X-ray diffraction}

PXRD profiles of GDCS1902 (Vendor 1 and Vendor 2) were collected using Bruker powder X-ray diffractometer (Model D8 ADVANCE). The X-ray is produced using the Copper anode $(\mathrm{K} \alpha 1, \lambda=1.5406 \AA$ ) and LynxEyeTM detector operating at a voltage of $40 \mathrm{kV}$ and current of $40 \mathrm{~mA}$. Furthermore, the diffraction pattern was collected in the 2-theta geometry (continuous mode), scan range $\left(3^{\circ}\right.$ to $\left.45^{\circ} 2 \theta\right)$, and step size of $0.01^{\circ} 2 \theta$ with a time per step of $0.1 \mathrm{sec}$. Samples were prepared using PMMA (Polymethyl methacrylate) sample holder ( $25 \mathrm{~mm}$ ) using the top load method. The acquired data were processed using Bruker Eva software.

\section{Raman spectroscopy}

All Raman spectra reported in this study were collected using an alpha 300 RA (WITec, Ulm, Germany) consisting of Zeiss microscope, coupled with a ultra-high throughput spectrometers and a Newton-EMCCD (Electron Multiplying Charge Coupled Device) camera (operating in standard mode with $1,600 \times 200$ pixels, at $-64^{\circ} \mathrm{C}$ with full vertical binning). The

Table 1. Physical properties of API (GDCS1902)

\begin{tabular}{ll}
\hline Property & Description \\
\hline Indications & $\begin{array}{l}\text { Antipsychotic drug } \\
\text { Yes (Form I and II , monotropic } \\
\text { relationship) }\end{array}$ \\
Polymorphism & $181-182^{\circ} \mathrm{C}$ \\
Melting point & 1.06 \\
Log P & 9.37 \\
pKa & $\sim 0.292 \mathrm{mg} / \mathrm{ml}$ \\
Intrinsic solubility & $400 \mathrm{mg}$ \\
Maximum dose & BCS 2B (Drug contains primary amine \\
Biopharmaceutical Classification System & group) \\
\hline BCS) based on 250 ml GI Fluids &
\end{tabular}


sample was irradiated using $785 \mathrm{~nm}$ laser with a $20 \times$ objective to the spot of $900 \mathrm{~nm}$ and the inelastic scattering was acquired in the backscattering geometry. The typical spectral resolution was set at $3 \mathrm{~cm}^{-1}$. The integration time of $2 \mathrm{~s}$ and the total accumulation of 10 was set as the spectrum collection parameters. The average laser intensity was $125 \mathrm{~mW}$. Spectra were acquired using WITec Project Plus software.

\section{Differential scanning calorimetry (DSC)}

The thermal properties of API from both the Vendors were analyzed using the DSC 2500 series (TA instruments, USA) differential scanning calorimeter. Approximately, $3.0 \mathrm{mg}$ of sample was weighed and sealed in Tzero pans with hermatic lid without any pinhole. The samples were equilibrated at $25^{\circ} \mathrm{C}$ and heated up to $200^{\circ} \mathrm{C}$ at a heating rate of $10^{\circ} \mathrm{C} /$ minute as well as $50^{\circ} \mathrm{C} /$ minute under nitrogen gas as purge atmosphere. The flow rate was set at $10 \mathrm{ml} /$ minute. The values of onset, endset, peak temperature, peak height ( $\mathrm{mJ}$ or $\mathrm{mW})$, peak area, and change in heat $(\mathrm{J} / \mathrm{g})$ for each peak were recorded.

\section{Polarized light microscopy (PLM)}

The polarized microscopic images were recorded with a charge-coupled device (CCD) camera attached to the Nikon LV100 POL microscope. The API samples from two different vendors were examined using PLM under various magnifications with one polar set-up. Morphology and particle size properties of both the samples were investigated.

\section{Fourier transform near-infrared (FT-NIR) spectroscopy}

Frontier FT-NIR spectrometer (PerkinElmer, Waltham, MA) fitted with a Near Infrared Reflectance Accessory equipped with a liquid nitrogen cooled Mercury Cadmium Telluride detector was employed for raw material and tablet analysis. Each spectrum was collected over the range of 1,000 to $2,500 \mathrm{~nm}$ (from 10,000 to $4,000 \mathrm{~cm}^{-1}$ ) at an average of 64 scans with a spectral resolution of $8 \mathrm{~cm}^{-1}$. Spectra from RM and tablets were collected using spectrum 10. The spectra were collected with the Spectrum Touch Software Version 10.0. Vials and Tablets were placed in a 26-mm aperture in the center. Five repeat measurements were collected per vial (for API) with random shaking and tapping between each measurement so as to determine any significant differences between the measurements/sample. The tablets consisted of embossing and break line. Since the depth of penetration of radiation is less than the thickness of the tablet, measurements on both the sides were taken into consideration, so as to rule out any variations.

\section{Chemometric analysis based on principal component analysis (PCA)}

The collected data were analyzed using Unscrambler 10.0 (CAMO Software AS, Oslo, Norway) software packages. PCA is used to derive interpretable information from a multidimensional space wherein the original variables are projected as a reduced number of latent variables termed as principal components (PCs). The end result is the conversion of the original data matrix to score plot which is called as eigenvalues (corresponding to concentration profile) and loading plot, termed as eigenvectors (corresponding to spectral profile). Plot of PCs provides interrelationship between the samples under investigation which then helps to interpret certain trends among samples, similarities, or dissimilarities between the investigated samples. In this study, the scatter plot will be utilized to derive a relationship between various samples like API and tablets from two vendors as well as between different prototype formulations.

\section{Sieve analysis}

The quantitative particle size distribution (PSD) analysis of API from two different vendors was determined using a vibratory sieve shaker AS200, Retsch, Haan, Germany. Using sieve mesh sizes of 45, 60, 80, 100, 120, 170, and pan. Approximately 100 $\mathrm{g}$ of "as received" API were weighed and then loaded on to the assembled sieves (ranging from pan to $400 \mu \mathrm{m}$ ). The experimental parameter adopted sieve shaking time of 5 min with an amplitude of vibration of $1.5 \mathrm{~mm} /$ “ $\mathrm{g}$ ”.

\section{RESULTS}

\section{X-ray diffraction (XRD)}

Figure 1 shows the PXRD pattern for the GDCS1902 from two different vendors. The diffraction pattern for the GDCS1902 from both the vendors is found to match with respect to peak position indicating that the API from both Vendors crystallize into the same polymorphic form. Both the RM showed some integrated intensity variations, for example, some of the reflections as indicated by the 2theta for API (Vendor 2) is very high as compared to Vendor 1. The origin of such observations and their implications are discussed in further sections.

\section{Raman spectroscopy}

The molecular-level properties of the materials are studied using Raman spectroscopy. Raman spectra are sensitive to crystal defects and amorphous content (Griffen et al., 2016). In this study, Raman spectra are used to measure if there are any crystallinity/amorphous content variations between the materials. The representative Raman spectra of GDCS1902 are shown in Figure 2A. The band appearing around $\sim 1,596 \mathrm{~cm}^{-1}$ is attributed to aromatic $\mathrm{C}=\mathrm{C}$ ring modes while the split peak appearing between 1,640 and $1,620 \mathrm{~cm}^{-1}$ is attributed to amide carbonyl. The other peaks of interest while comparing the APIs from two different vendors are the $\mathrm{CH}_{2}$ bending mode falling in the region between

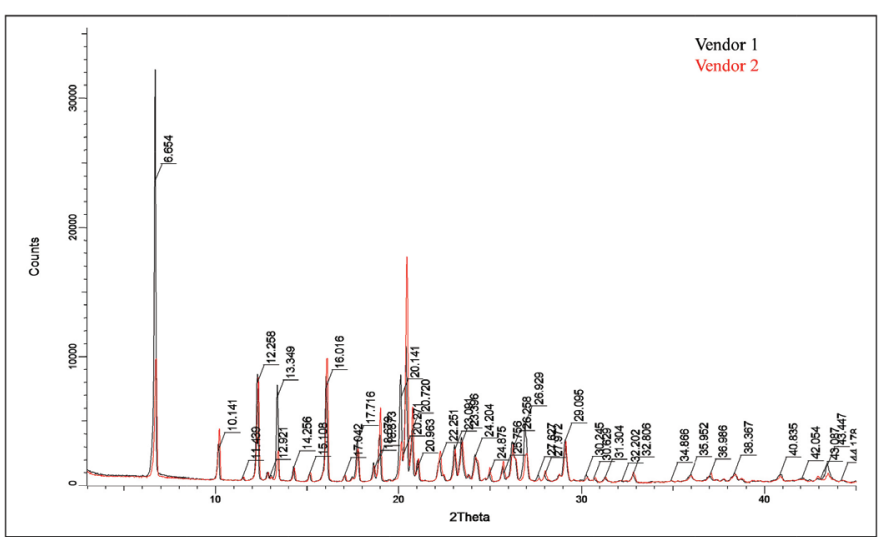

Figure 1. Representative XRD diffractogram for API from Vendor 1 and Vendor 2 (GDCS1902). 


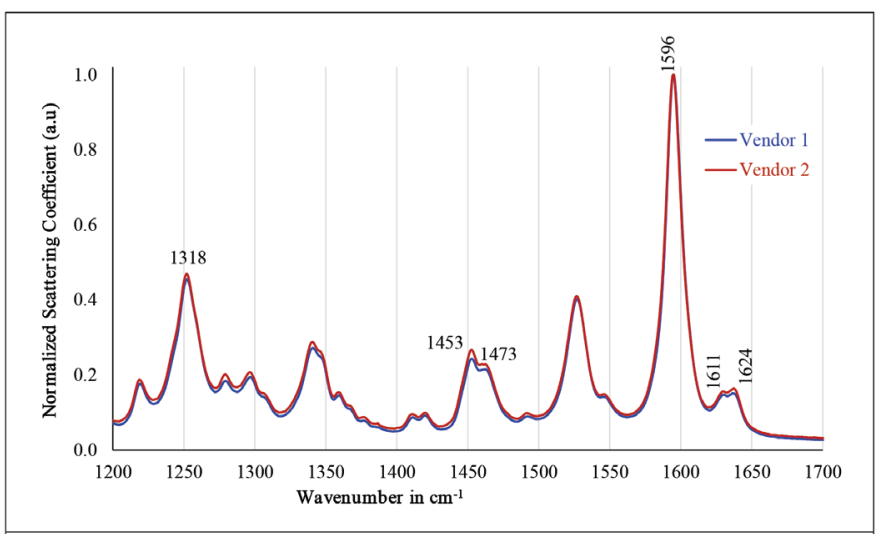

(a)

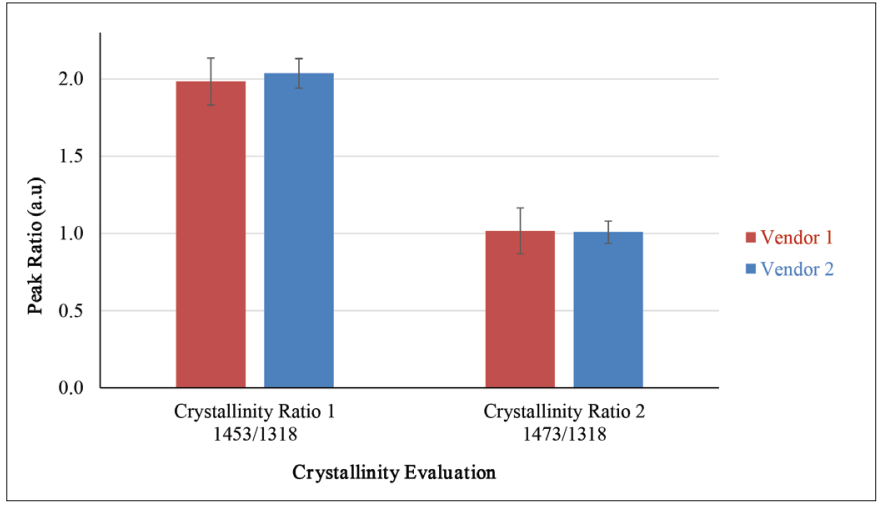

(b)

Figure 2. (A) Typical Raman spectra of various API (GDCS1902) particles. (B) Relative crystallinity estimation for API (GDCS1902) from Vendor 1 and Vendor 2 by means of Raman spectra (the error bars are measurement of six spectra collected from various particles of differing morphologies, $n=6$ ).

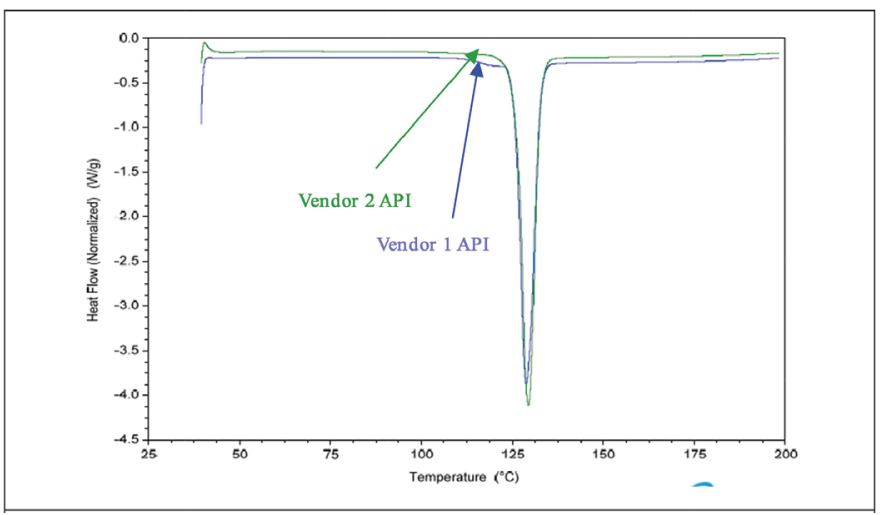

Figure 3. DSC analysis of API from Vendor 1 and Vendor 2 at $10^{\circ} \mathrm{C} /$ minute and $50^{\circ} \mathrm{C} /$ minute (GDCS1902).

1,440 and $1,480 \mathrm{~cm}^{-1}$. These peaks are frequently applied in the polymer and pharmaceutical industry to evaluate the crystallinity of the material (Lehto et al., 2006). There are also Raman bands appearing around $\sim 1,318 \mathrm{~cm}^{-1}$ which can be assigned to $\mathrm{CH}_{2}$ twisting modes (Lehto et al., 2006; Petzoldt et al., 2014). This band can be used as an internal standard to understand the crystallinity/ amorphicity and molecules' orientation. The ratio between $\mathrm{CH} 2$ bending and twisting mode $\left(1,473 / 1,318 \mathrm{~cm}^{-1}, 1,453 / 1,318 \mathrm{~cm}^{-1}\right)$ shows the nature of APIs from both the vendors (Fig. 2B).

\section{Differential scanning calorimetry (DSC)}

Figure 3 shows the DSC thermograms of API from Vendor 1 and Vendor 2. Both the APIs displayed peak melting around $129^{\circ} \mathrm{C}$. The thermogram similarity essentially confirms that there is no polymorphic change and these results are in line with XRPD. Moreover, no degradation in the melting region for both the API samples is expected as no shift in the baseline is observed. However, API from Vendor 1 displays a faster onset of the melting-like event which is not observed with API from Vendor 2. Since the API displays monotropic polymorphic relationship, the origin of this peak is discussed in detail in the following sections.

\section{Polarized light microscopy (PLM)}

Figure 4A-C and 4D-F show examples of PLM images displaying the morphology of GDCS1902 from Vendor 1 and Vendor 2, respectively. Analysis of API particle shape is deemed very crucial to discriminate between Vendors. The Various shapes observed with API (Vendor 1) are squarish, long rectangles, triangles, etc., whereas the particle shape of API from Vendor 2 is quite uniform and consists of medium rectangles. Also, fine particles are observed with Vendor 1 but not with Vendor 2. Therefore the particulate properties for both the vendors are found to be dissimilar and the impact of such properties on product performance has been subject of many research studies.

\section{NIR spectroscopy and chemometric analysis}

NIR spectroscopy is a powerful solid-state characterization tool that measures the molecular-level properties of the materials (Chavan et al., 2017). It is one of the very few techniques that are available to probe the physical and chemical properties of the material in a single measurement (Khorasani et al., 2016). NIR spectra capture the physical and chemical properties of the samples; however, untying the physical property contributions from chemical contributions requires the use of chemometric tools (Chavan et al., 2017; Talwar et al., 2017). From orthogonal solid state tools, it is established that there is an absence of any evidence for API transformation or crystal disorders or amorphization etc. NIR spectroscopy was used to measure if any variations between the materials supplied by two different vendors, which could be then correlated to particle size or morphological variations in line with PLM measurements. The spectra of API from two vendors are acquired and displayed in Figure 5A. The absorption peaks at $8,532,6,788,6,030-5,685$, and 4,968-4,320 $\mathrm{cm}^{-1}$ are associated with aromatic $(\mathrm{CH}=\mathrm{CH})$, overtone of amine $\left(\mathrm{NH}, \mathrm{NH}_{2}\right)$, overtones of $\mathrm{C}-\mathrm{H}$ vibrations from aromatic, aliphatic chains, and combination bands arising from $\mathrm{CH}$ and carbonyls, respectively. As expected, no new peaks or absence of any peaks between the vendors are observed, indicating no polymorphic or crystallinity variations between the two APIs. In order to capture the physical property variations, PCA projection and PC interpretation of the score plot of the two vendors without any pre-treatment of data was carried out. To interpret physical effects between the two samples, PCA scatterplot using two PCs were used (Fig. 5B). 


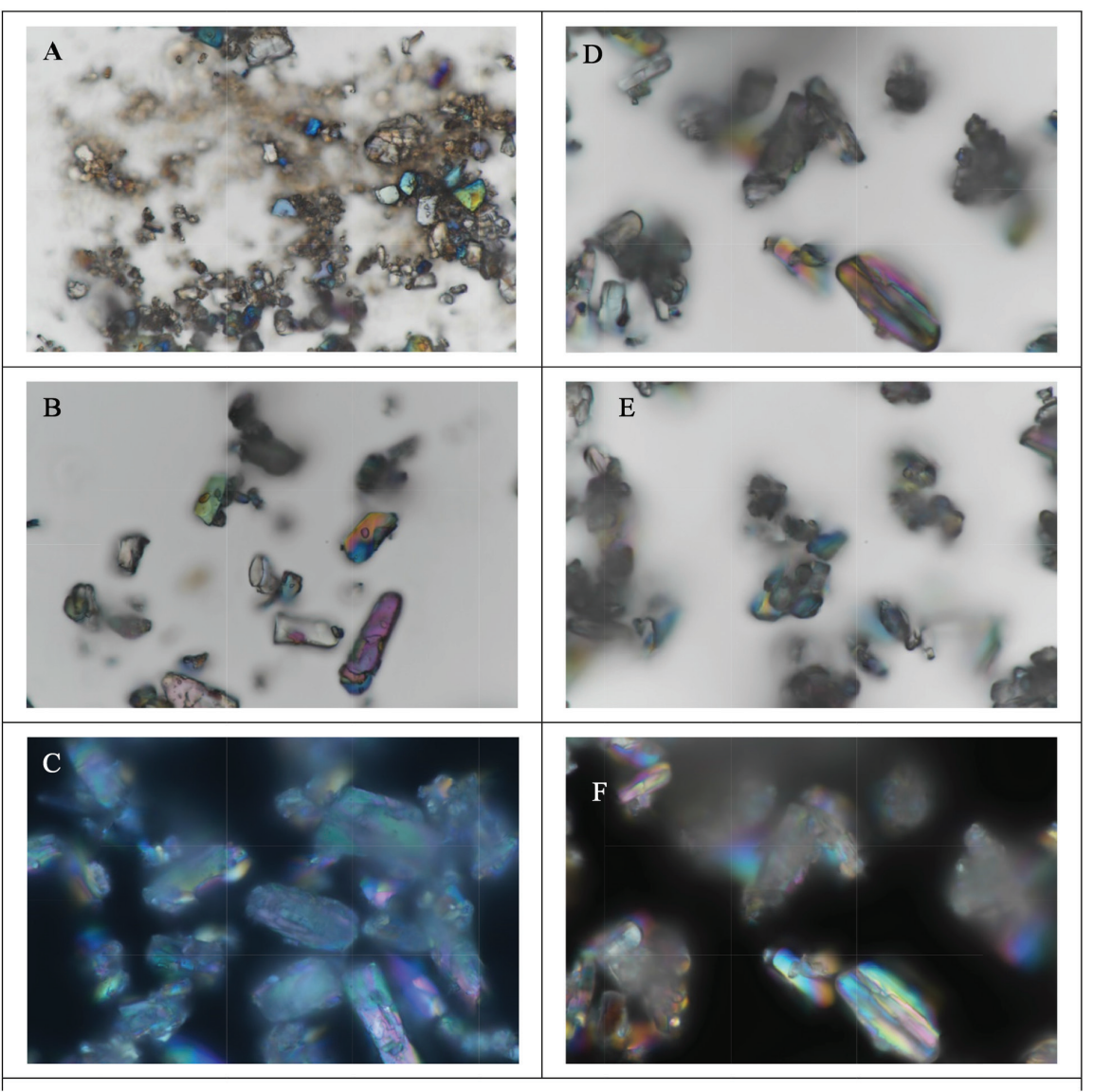

Figure 4. Polarized light microscopy images (both brightfield and darkfield) of API from Vendor 1 (A-C) and Vendor 2 (D-F) (GDCS1902) at 50× magnification.

\section{Sieve analysis}

Particle size determination employing sieves with different-sized mesh is considered to be one of the oldest, reliable, quick, least expensive, representative of lots, and most commonly used methods. Particle size distribution of the examined APIs is tabulated in Table 2. Histogram plot of the \% mass fraction retained as a function of particle size for two vendors is shown in Figure 6. Three size fractions were compared between the vendors: (1) particles $<149 \mu \mathrm{m}$, (2) particles equivalent to $149 \mu \mathrm{m}$, and (3) particles $>149 \mu \mathrm{m}$.

\section{DISCUSSION}

\section{Characterization of the API from different vendors}

\section{Polymorphic investigation- PXRD}

GDCS1902 displays polymorphism and are designated as form I and form II (monotropic polymorphism) (see Table 1). Figure 1 compares the PXRD pattern of GDCS1902 from two different vendors. The integrated peak position of GDCS1902 from both the vendors was found to match and therefore it was concluded that GDCS1902 from these vendors consist of form I showing that there is no PIP. Moreover, the absence of halo pattern from both the vendors indicates the absence of PICD concluding that the API manufacturing process from both the vendors did not induce any phase transformations or crystal defects. However, integrated peak intensity variations are observed between these APIs. In general, such observations could be attributed to particle size or morphological differences (Arsoy et al., 2017). In order to further investigate the PIF orthogonal tools like thermal analysis, microscopy, and spectroscopy were utilized.

Surface molecular composition measurements to evaluate crystallinity-Raman spectroscopy

Raman spectroscopy has been widely employed and has an advantage over other techniques to detect low percentages of both crystalline and amorphous components in a sample. The plot of peak intensity ratios (crystallinity ratio 1,2, and 3) for the APIs from a different vendor can be compared to evaluate the crystallinity variations between the samples. The results of these were found to be identical. Based on these results, it is interpreted that there are no significant differences observed between the two vendors with respect to crystallinity/amorphicity fractions. These results are in line with the PXRD measurements.

\section{Bulk properties evaluation-differential scanning calorimetry} (DSC)

Both the APIs display peak melting peak around $\sim 129^{\circ} \mathrm{C}$. The curve for API (Vendor) was found to be steeper than the Vendor 1. The inflection max point for API (Vendor 1) was $115^{\circ} \mathrm{C}$ and $124^{\circ} \mathrm{C}$ for Vendor 2. The faster onset of melting observed with API (Vendor 1) is attributed to concentrations of varied-sized 


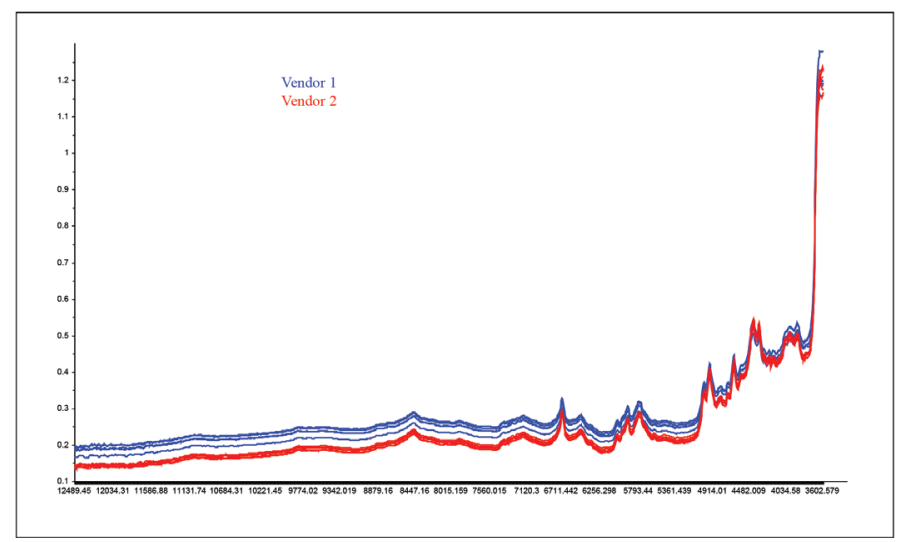

(a)

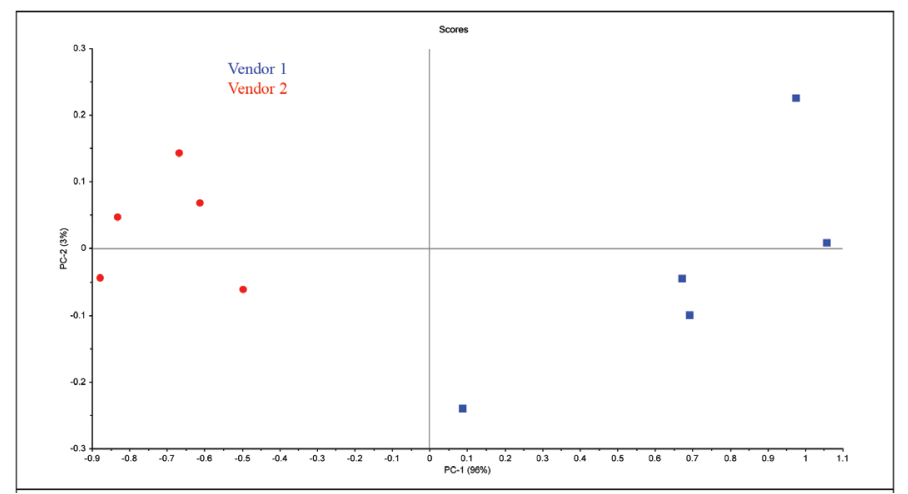

(b)

Figure 5. (A) NIR spectra of repeated measurements of API (GDCS1902) from Vendor 1 and Vendor 2. (B) Representative PCA score scatterplot for API (GDCS1902) from two vendors.

Table 2. PSD of API (GDCS1902) from two different vendors.

\begin{tabular}{cccccc}
\hline Sieve no & $\begin{array}{c}\text { Particle size } \\
\text { distribution } \\
\text { (in } \boldsymbol{\mu m})\end{array}$ & $\begin{array}{c}\text { Vendor 1 (\% mass } \\
\text { fraction retained) }\end{array}$ & $\begin{array}{c}\text { Vendor 2 (\% mass } \\
\text { fraction retained) }\end{array}$ \\
\hline $45,60,80$ & $>149$ & $13 \%$ & $24 \%$ & $59 \%$ & $75 \%$ \\
100 & 149 & $11 \%$ & $16 \%$ & \multicolumn{2}{c}{$25 \%$} \\
$\begin{array}{c}120,170, \\
\text { Pan }\end{array}$ & $<149$ & $76 \%$ & & \multicolumn{2}{c}{$25 \%$} \\
\hline
\end{tabular}

particles. The steeper and sharper onset of melting observed with API (Vendor 2) API indicates a narrow spectrum or uniformsized particles. The differences in the onset of melting indicate that the process employed for the manufacture of GDCS1902 by both the Vendors is different. Vendor 1 is assumed to use a slightly high shear process than Vendor 2 due to which more nonuniform particles were observed. The differences in the range of particle sizes and/or morphology undoubtedly impact the melting behavior (Van Dooren et al., 1982). To further provide evidence on the process-induced fragmentation, morphological analysis using PLM was carried out.

\section{Powder properties and morphology-PLM}

PLM images indicate the 2 crystalline nature of GDCS1902 and the results are in-line with XRD. Additionally, API from Vendor 2 was found to be better dispersed, separated,

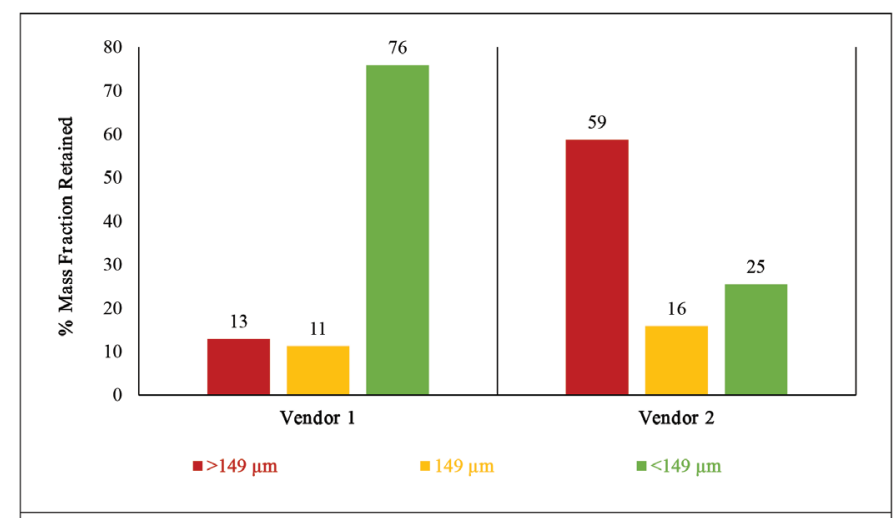

Figure 6. Particle size distribution (analytical sieve analysis) of API (GDCS1902) from two different vendors.

and existed as primary particles than Vendor 1 . The Vendor 1 API is found to be platy, flat, medium rectangle, and uniform, whereas Vendor 2 API particles display varied crystal shapes, such as platy, flat, square, and triangle. Selection of the milling and/or micronization process parameters can advertently affect the particle size and morphology of rod- or needle- or rectangleshaped APIs (Ferreira et al., 2018; Schenck et al., 2019; Stauffer et al., 2019). This observation further confirms the results obtained from DSC and XRD.

\section{Molecular properties-NIR}

From the orthogonal solid-state characterization, it is clear that the two APIs from different vendors are chemically and polymorphically identical. Also, the crystallinity of API from both vendors also is equivalent. The FT-NIR spectra of these API also demonstrate that these samples are identical. However, spectral baseline slope/offsets were observed, which can be attributed to the particle size effect (Sarraguca et al., 2011). Further, it is well established that the NIR spectral baseline offset is inversely proportional to particle size, that is, the spectral baseline offset increases with decreasing the particle size (Schwanninger et al., 2011). From this, it can be interpreted that API from Vendor contains more fine particles than the Vendor 2 which is seen with both, NIR spectra and with PCA scatterplot. Figure 5B shows that using the PCA model, total variations between the two vendors could be explained using two PCs. Well-separated two different clusters representing Vendor 1 and Vendor 2 are obtained. Although equivalent with respect to compendial tests, polymorphic test, and crystallinity tests, these materials are not equivalent with respect to particulate properties. These results are also in line with DSC and PLM results. Considering this, changes to the formulation process such as process-induced fragmentation was carried out for API from Vendor 2

\section{Sieve analysis}

From figure 6, it is evident that both the vendors display dissimilarity in the particle size fractions. Approximately $75 \%$ of API particles from Vendor 1 are $<149 \mu \mathrm{m}$, whereas Vendor 2 is much $>$ $149 \mu \mathrm{m}$, which indicates the presence of more small particles in API from Vendor 1. These results are in line with PLM and NIR results. Hence, a modification in the formulation process was performed. 


\section{Formulation changes}

To further evaluate the hypothesis, change in formulation process was taken into consideration API from Vendor 2 with respect to binder addition and kneading. Here the granulation process was changed to induce higher shear by increasing impeller and chopper speed, which is required to achieve desired granule properties by decreasing the particle size. This process changes led to the fragmentation that produced fine particle equivalent to Vendor 1. The prototype formulations were then subjected to drug product performance testing and compared for disintegration time and single-point dissolution (data not shown). Prototype 1 and new Prototype (Prototype 3) formulations were found to comply with the in-house specification, which is, disintegration time of $<4.5$ mins and $>70 \%$ drug substance release in 45 minutes. In conclusion, the integration of API critical material attributes with manufacturing process enabled to overcome API sourcing difficulties from multiple vendors.

\section{CONCLUSION}

Despite the fact that model API from two different sources met the compendial requirements and related quality control tests, the final product performance in tablet formulation did not comply with the specification. To overcome such experiences, more emphasis should be given to establish critical material attributes that are related to functional characteristics, to qualify alternate vendors.

XRD, Raman studies showed that vendormanufacturing process did not induce any polymorphic transformations or crystal disorder. Since the API is needleshaped or rod-shaped particles, the other mechanism that could be inferred from microscopy and thermal analysis was differences in the process-induced fragmentation. The physical properties like morphology, small particulate fractions, and particle shape that differed significantly in both the Vendors were found to be CMAs. Therefore, solid-state tools were employed to understand these CMAs and also process/formulation change such as an increase in granulation stress was applied to circumvent product failure. The understanding of CMAs and process/formulation changes resulted in compliance with the dissolution test for Prototype 3. The current study highlights the importance of providing unprecedented knowledge as well as interfacing the raw material attributes with that of the pharmaceutical processing parameters which would help in designing a robust product with minimal variability. To design a robust formulation and process, understanding the causes of raw material variability is very crucial. In this regard, the selection of right analytical and solid-state characterization tools is deemed imperative. Model raw material qualification system to ensure consistency of the material from lot to lot or vendor to vendor, sufficing early product development, late-stage development, life cycle management requirements, API supplier market recalls, quality or vendor GMP facility observations/warning letters, and alternate API supplier adoption, etc., are established.

\section{ACKNOWLEDGMENTS}

The authors are so much obliged to Sanofi and Dr. Praveen Khullar, Head of Global Development Centre, Sanofi
Synthelabo (India) Private Limited, Goa and Dy. Head, Scientific and Technical Services for providing the facilities to carry out the aforementioned work. The authors would like to thank Dr. A. Rose Venis, Associate Professor, Department of Chemistry, St. Joseph's College, Tiruchirappalli for providing guidance and needful support.

\section{CONFLICTS OF INTEREST}

All authors declare that there are no conflicts of interest.

\section{REFERENCES}

Arsoy Z, Ersoy B, Evcin A, Icduygu MG. Influence of dry grinding on physicochemical and surface properties of talc. Physicochem Probl Miner Process, 2017; 53:288-306.

Byrn SR, Zografi G, Chen S. Solid state properties of pharmaceutical materials. John Wiley \& Sons, New Jersey, 2017.

Carlton RA. Pharmaceutical microscopy. Springer Science \& Business Media, New York, NY, 2011.

Chavan RB, Bhargavi N, Lodagekar A, Shastri NR. Near infrared spectroscopy: a tool for solid state characterization. Drug Discov Today, 2017; 22:1835-43.

Chennuru R, Muthudoss P, Voguri RS, Ramakrishnan S, Vishweshwar P, Babu RR, Mahapatra S. Iso-structurality induced solid phase transformations: a case study with lenalidomide. Cryst Growth Des, 2016; 17:612-28.

Descamps M, Willart JF. Perspectives on the amorphization milling relationship in pharmaceutical materials. Adv Drug Delivery Rev, 2016; 100:51-66.

Docherty R, Kougoulos T, Horspool K. Materials science and crystallization: the interface of drug substance and drug product. Am Pharm Rev, 2009; 12:34-43.

Elisei E, Willart JF, Danède F, Siepmann J, Siepmann F, Descamps M. Crystalline polymorphism emerging from a milling-induced amorphous form: the case of chlorhexidine dihydrochloride. J Pharm Sci, 2018; 107:121-6.

Elder DP, Kuentz M, Holm R. Pharmaceutical excipientsquality, regulatory and biopharmaceutical considerations. Eur J Pharm Sci, 2016; 87:88-99.

Ferreira AP, Gamble JF, Leane MM, Park H, Olusanmi D, Tobyn M. Enhanced understanding of pharmaceutical materials through advanced characterisation and analysis. AAPS PharmSciTech, 2018; 19:3462-80.

Giron D. Thermal analysis of drugs and drug products, In: Swarbrick J (ed.). Encyclopedia of pharmaceutical science and technology, Six Volume Set. CRC Press, Boca Raton, FL, pp 3591-614 , 2013.

Griffen JA, Owen AW, Burley J, Taresco V, Matousek P. Rapid quantification of low level polymorph content in a solid dose form using transmission Raman spectroscopy. J Pharm Biomed Anal, 2016; 128:35-45.

Ho R, Naderi M, Heng JY, Williams DR, Thielmann F, Bouza P, Keith AR, Thiele G, Burnett DJ. Effect of milling on particle shape and surface energy heterogeneity of needle-shaped crystals. Pharm Res, 2012; 29:2806-16.

Kandpal LM, Park E, Tewari J, Cho BK. Spectroscopic techniques for nondestructive quality inspection of pharmaceutical products. J Biosyst Eng, 2015; 40:394-408.

Khorasani M, Amigo JM, Bertelsen P, Sun CC, Rantanen J Process optimization of dry granulation based tableting line: extracting physical material characteristics from granules, ribbons and tablets using near-IR (NIR) spectroscopic measurement. Powder Technol, 2016; 300:120-5.

Kushner IV J. Utilizing quantitative certificate of analysis data to assess the amount of excipient lot-to-lot variability sampled during drug product development. Pharm Dev Technol, 2013; 18:333-42.

Lehto VP, Tenho M, Vähä-Heikkilä K, Harjunen P, Päällysaho M, Välisaari J, Niemelä P, Järvinen K. The comparison of seven different methods to quantify the amorphous content of spray dried lactose. Powder Technol, 2006; 167:85-93. 
Moros J, Garrigues S, de la Guardia M. Vibrational spectroscopy provides a green tool for multi-component analysis. Trends Analyt Chem, 2010; 29:578-91.

Newman A, Wenslow R. Solid-state characterization techniques, In: Li T, Mattei A (eds.). Pharmaceutical crystals. Science and Engineering, John Wiley \& Sons Inc., New Jersey, pp 89-121, 2018.

Pasquini C, Near infrared spectroscopy: a mature analytical technique with new perspectives-a review. Anal Chim Acta, 2018; 1026 $8-36$.

Paudel A, Raijada D, Rantanen J. Raman spectroscopy in pharmaceutical product design, pharmaceutical applications of Mid-IR and Raman spectroscopy. Adv Drug Delivery Rev, 2015; 15:3-20.

Petzoldt C, Bley O, Byard SJ, Andert D, Baumgartner B, Nagel N, Tappertzhofen C, Feth MP. An example of how to handle amorphous fractions in API during early pharmaceutical development: SAR114137-a successful approach. Eur J Pharm Biopharm, 2014; 86:337-50.

Sarraguça MC, Cruz AV, Amaral HR, Costa PC, Lopes JA. Comparison of different chemometric and analytical methods for the prediction of particle size distribution in pharmaceutical powders. Anal Bioanal Chem, 2011; 399:2137-47.

Schenck L, Koynov A, Cote A. Particle engineering at the drug substance, drug product interface: a comprehensive platform approach to enabling continuous drug substance to drug product processing with differentiated material properties. Drug Dev Ind Pharm, 2019; 45:521-31.

Schwanninger M, Rodrigues JC, Fackler K. A review of band assignments in near infrared spectra of wood and wood components. J Near Infrared Spectrosc, 2011; 19:287-308.

Stauffer F, Vanhoorne V, Pilcer G, Chavez PF, Rome S, Schubert MA, Aerts L, De Beer T. Raw material variability of an active pharmaceutical ingredient and its relevance for processability in secondary continuous pharmaceutical manufacturing. Eur J Pharm Biopharm, 2018; 127:92-103.

Stauffer F, Vanhoorne V, Pilcer G, Chavez PF, Schubert MA, Vervaet C, De Beer T. Managing active pharmaceutical ingredient raw material variability during twin-screw blend feeding. Eur J Pharm Biopharm, 2019; 135:49-60.
Talwar S, Roopwani R, Anderson CA, Buckner IS, Drennen JK Determination of spatially resolved tablet density and hardness using nearinfrared chemical imaging (NIR-CI). Appl Spectrosc, 2017; 17:1906-14.

Trasi NS, Boerrigter SX, Byrn SR. Investigation of the millinginduced thermal behavior of crystalline and amorphous griseofulvin. Pharm Res, 2010; 27:1377-89.

Van Dooren AA, Müller BW. Effects of heating rate and particle size on temperatures and specific enthalpies in quantitative differential scanning calorimetry. Thermochim Acta, 1982; 54:115-29.

Zarmpi P, Flanagan T, Meehan E, Mann J, Fotaki N. Biopharmaceutical aspects and implications of excipient variability in drug product performance. Eur J Pharm Biopharm, 2017; 111:1-5.

Zhao C, Jin C, Gao H, Wang L, Liu H, He Z. Effect of raw material variability of glipizide on the in vitro dissolution rate and in vivo bioavailability performance: the importance of particle size. AJPS, 2019; $14: 165-73$

Zimper U, Aaltonen J, McGoverin C, Gordon KC, KrauelGoellner K, Rades T. Quantification of process induced disorder in milled samples using different analytical techniques. Pharmaceutics, 2010; 2:30-49.

How to cite this article:

Saravanan D, Muthudoss P, Khullar P, Rosevenis A. Vendor qualification: Utilization of solid state characterization "Toolbox" to assess material variability for active pharmaceutical Ingredient. J Appl Pharm Sci, 2019; 9(09):001-009. 


\section{GRAPHICAL ABSTRACT}

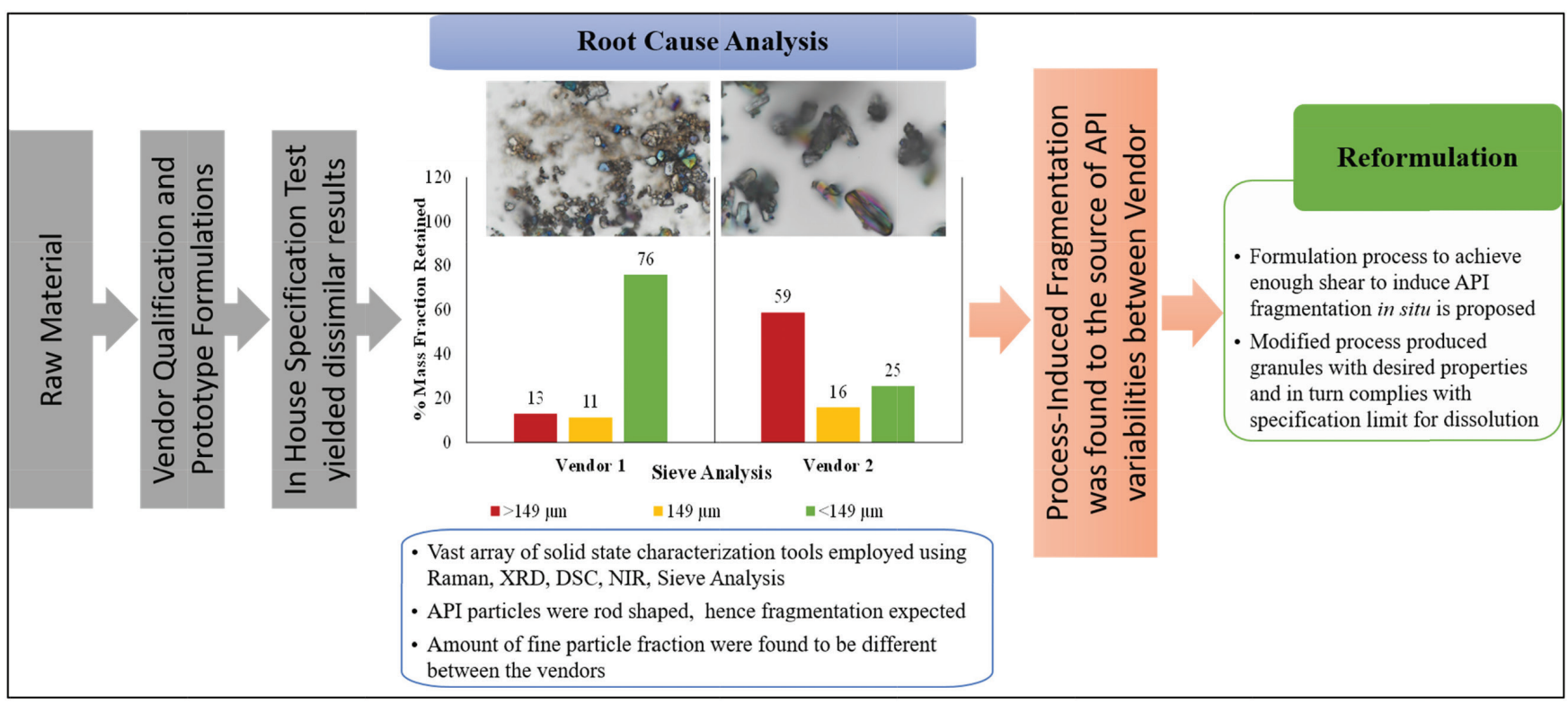

Process-Induced Fragmentation was found to be the source of API variability between Vendors 